\title{
The Relationship Between Type D Personality and the Complexity of Coronary Artery Disease
}

This article was published in the following Dove Press journal:

Neuropsychiatric Disease and Treatment

\author{
Virgil Radu Enatescu (DD ${ }^{1, *}$ \\ Dragos Cozma ${ }^{2,3, *}$ \\ Diana Tint ${ }^{4,5}$ \\ Ileana Enatescu ${ }^{6}$ \\ Mihaela Simu' \\ Catalina Giurgi-Oncu (D) \\ Mihai Andrei Lazar ${ }^{2}$ \\ Cristian Mornos ${ }^{2,3}$ \\ 'Department of Neuroscience, "Victor \\ Babes" University of Medicine and \\ Pharmacy Timisoara, Timisoara, Romania; \\ ${ }^{2}$ Department of Cardiology I, "Victor \\ Babes" University of Medicine and \\ Pharmacy Timisoara, Timisoara, Romania; \\ ${ }^{3}$ Institute of Cardiovascular Diseases \\ Timisoara, Timisoara, Romania; ${ }^{4}$ School \\ of Medicine, Transylvania University, \\ Brasov, Romania; ${ }^{5}$ Department of \\ Cardiology, ICCO Clinics Brasov, Brasov, \\ Romania; ${ }^{6}$ Department of Obstetrics and \\ Gynecology-Discipline of Childcare and \\ Neonatology, "Victor Babes" University \\ of Medicine and Pharmacy Timisoara, \\ Timisoara, Romania
}

*These authors contributed equally to this work

\begin{abstract}
Purpose: The relationship between personality traits and cardiovascular disease has gathered sustained interest over the last years, type -D personality (TDP) being significantly associated with coronary artery disease (CAD). However, data regarding the connection between the TDP and the severity of CAD disease is scarce. The aim of our study was to assess the relationship between TDP and the complexity of CAD, and to compare it with other sociodemographic and clinical features.
\end{abstract}

Patients and Methods: We conducted a cross-sectional case-control clinical-based study on 221 consecutive hospitalized patients with chest pain (60 \pm 10.2 years; 131 men), referred for coronary angiography.

Results: TDP was identified in 42 (19\%) patients, using the DS 14 scale. Symptomatology profile was evaluated using the SCL-90 scale. Syntax score was greater in the subgroup of patients with TDP in comparison to non-TDP subgroup $(26.21 \pm 12.03$ vs $15.49 \pm 8.89$, respectively, $\mathrm{p}<0.001$ ), and most of SCL-90 symptom dimensions have significantly higher levels in the subgroup of TDP with CAD patients (all $p<0.05$ ). Smoking $(\beta=0.132, p=0.037$ ), dyslipidemia $(\beta=0.149, \mathrm{p}=0.013)$, Diabetes Mellitus $(\beta=232, \mathrm{p}<0.001)$, NA dimension of TDP $(\beta=0.255, \mathrm{p}<0.001)$ and SI $(\beta=0.279, \mathrm{p}<0.001)$ dimension of TDP have a significant contribution to the complexity of CAD assessed by Syntax score.

Conclusion: TDP was associated with a more complex CAD assessed by Syntax score, and may represent a dynamic interface between the biological and psychological vulnerabilities and the symptoms of CAD.

Keywords: coronary artery disease, type D personality, Syntax score

\section{Introduction}

Cardiovascular diseases continue to represent the leading cause of death worldwide, with being responsible for $45 \%$ of all deaths in Europe. Moreover, coronary heart disease by itself accounts for about $20 \%$ of all deaths in Europe annually, thereby surpassing even breast cancer in women and lung cancer in men. ${ }^{1,2}$

The relationship between cardiac diseases and different psychological features of the affected individuals should be regarded as more complex and bidirectional. Recently, the advances in research of cardiac diseases have led to the conclusion that depression should be recognized as a risk factor for poor prognosis in patients with acute coronary syndrome. ${ }^{3}$ Thus, it was the first instance when a psychological factor was weighted to the same extent as other previously known traditional risk factors such as hypertension, family history, hypercholesterolemia, diabetes mellitus (DM) and smoking. ${ }^{4,5}$
Correspondence: Ileana Enatescu Email enatescu.ileana@umft.ro

Diana Tint

Email dianatint@gmail.com

Neuropsychiatric Disease and Treatment 2021:17 809-820 
On the other hand, some of the personality traits that are more stable could play a more significant concurrent pathogenic role over time in coronary heart disease, rather than depression, which is considered as a temporary altered state of mood. Since the 1950s, there have been concerns related to identifying particular personality profiles and to correlate them with certain types of chronic medical conditions. Hence, Friedman and Rosenman's contribution is a turning point concerning research on the role of personality in CAD. ${ }^{6}$ More recently, the type-D or "distressed" personality concept was conceived by Denollet to detect those traits that place individuals at increased risk of CAD. The type D personality (TDP) consists of two sub-dimensions, namely social inhibition (SI) and negative affectivity (NA), where NA refers to the tendency to experience feelings of dysphoria, anxiety, and irritability, while SI refers to the tendency to inhibit the expression of emotions or behaviors in social interactions to avoid disapproval by others. It has been noticed that the TDP is significantly associated with cardiovascular diseases, but also with diabetes mellitus (DM). ${ }^{7}$ The TDP has elicited the researcher's interest, both as a vulnerable terrain for the heart disease occurrence and as a worsening factor that negatively impacts health outcomes of cardiovascular diseases, and especially outcomes following myocardial infarction. ${ }^{8,9}$ However, a few studies have found no associations between Type D personality and adverse cardiovascular outcomes. ${ }^{10,11}$

The aim of our study was to assess the relationship between TDP and the complexity of CAD, and to compare it with other sociodemographic and clinical features, assessing the hypothesis that TDP could negatively impact CAD reflected by the Syntax score.

\section{Patients and Methods}

\section{Procedure}

A cross-sectional case-control clinical-based study was conducted in consecutive patients with chest pain and age of at least 18 referred to the Institute of Cardiovascular Diseases Timisoara for coronary angiography according to current guidelines: disabling stable angina pectoris (CCS III-IV) despite medical therapy, high-risk criteria on noninvasive testing, angina in conjunction with signs of heart failure, recurrence of angina after PCI/CABG, survivors of cardiac arrest, severe ventricular arrhythmias, inconclusive or conflicting results after noninvasive stress testing, unable to undergo noninvasive testing (disability, illness, or morbid obesity), acute coronary syndrome.

All study participants were on appropriate medical therapy and underwent anamnesis, clinical examination, 12-lead electrocardiogram, blood samples, transthoracic echocardiogram, and coronary angiography, followed by psychological tests. The study was approved by Ethics Commission in Research - Development of the Institute of Cardiovascular Diseases Timisoara (registration number: 1658/28 March 2014). This study was conducted in accordance with the Declaration of Helsinki. Informed written consent was obtained from all patients.

\section{Sociodemographic, Case History and Major Risk Factors}

Sociodemographic data were collected as follows: current age, gender, educational level, residency, professional and marital status. Concerning case history data and biological data, the heredity for cardiovascular diseases, hypertension, hypercholesterolemia, diabetes mellitus, smoking and unharmful current alcohol drinking (a drink is equivalent with 14 grams of pure alcohol; so, moderate drinking is defined as up to 1 drink per day for women and up to 2 drinks per day for men), were recorded according to current guidelines. ${ }^{12,13}$

\section{Echocardiography}

Echocardiography was performed with a Vivid 9 ultrasonographic system (General Electric, Milwaukee, WI), in accordance with guidelines. ${ }^{14,15}$ All images were digitally stored and analyzed off-line with EchoPac PC Dimension software (GE Medical). Left ventricular ejection fraction (LVEF) was calculated from apical two- and four-chamber views using LV volumes by the modified biplane Simpson rule. ${ }^{14}$ Peak early (E) and E-velocity deceleration time were measured from the LV-inflow pattern at the tips of the mitral valve at end-expiratory time. ${ }^{15}$

Peak systolic (S') and peak early diastolic velocities (E') were obtained at the septal and lateral sites of the mitral annulus by pulse wave tissue Doppler imaging from apical four-chamber views. All velocities were recorded for three consecutive cardiac cycles during end-expiratory apnea, and the results were averaged. The E/E' ratio using the average of the septal and lateral E' was calculated. ${ }^{16,17}$

Speckle tracking echocardiography was also used for myocardial deformation measurements. Longitudinal peak systolic strain was determined from apical planes (four-, 
three- and two-chamber view) in a $17 \mathrm{LV}$ segment model. Value of global longitudinal strain (GLS) was obtained by averaging all $17 \mathrm{LV}$ segments. ${ }^{18,19}$ Off-line analysis was performed by two observers, who were blinded to clinical data.

\section{Coronary Angiography}

Coronary angiography results were evaluated by an interventional cardiologist, who was blinded to the psychological tests. The complexity of CAD was determined by the SYNTAX score, a tool which takes into consideration the anatomical features of the coronary lesions. ${ }^{20,21}$ The SYNTAX score is the sum of the points assigned to each individual lesion identified in the coronary tree with $>50 \%$ narrowing in the diameter of the vessels that measure greater than $1.5 \mathrm{~mm}$.

\section{Type D Personality}

The DS 14 scale was used to assess TDP. This scale comprises 14 items, each item being scored between $0=$ false and $4=$ true, and consists of two subscales representing two distinct dimensions of TDP (Negative Affectivity and Social Inhibition). A score $\geq 10$ on each subscale is required to meet the $\mathrm{D}$ personality. ${ }^{22,23}$ Moreover, the DS14 was shown to have a good reliability in an extensive cross-cultural equivalence analysis including most European countries and English-speaking countries, except Russia, with a Cronbach's $\alpha$ between 0.80 and 0.90 for NA and between 0.74 and 0.89 for $\mathrm{SI}^{24}$

\section{SCL-90 (Symptom Check List - 90)}

The SCL-90 is a multidimensional self-assessment inventory consisting of 90 items that cover nine symptomatology domains, such as somatization, obsessive-compulsive, interpersonal sensitivity, depression, anxiety, hostility, phobic anxiety, paranoid ideation, and psychoticism. Each item is scored on a Likert scale varying between $0=$ not at all to 4 = extremely. The SCL-90 scale is not a diagnostic tool; thus, it only measures a symptomatologic profile over the previous seven days before the self-assessment. ${ }^{25}$

\section{Participants}

Of 250 initially eligible patients for our study, 12 denied participating in the research and 17 patients were excluded due to missing data. Finally, after the inclusion and exclusion criteria were applied, a total number of 221 hospitalized subjects were enrolled to participate in the study. Patients with positive past or current personal history for any psychiatric disorders, including alcohol or other psychoactive use disorders, the absence of a signed informed consent, confusional state or any mental ability to provide written informed consent, inadequate echocardiographic images, paced rhythm, mitral prosthesis, congenital heart disease, renal failure (defined as a serum creatinine $>2 \mathrm{mg}$ / $\mathrm{dL}$ ), thyroid disorders, acute infections, stroke, or diabetic ketoacidosis, and age below 18 were not included in the study group.

The used method of sampling to enroll patients was by convenience including consecutive patients in order to increase the sample size. Enrolled subjects were provided with the self-assessment scales, indicated in the measures section below. Patients were stratified based on the presence or absence of TDP.

\section{Statistical Analyses}

Gender, family history of cardiovascular diseases, dyslipidemia, DM and smoking were recorded as dichotomous variables. The staged preexisting comorbid hypertension was quantified by using the ordinal variable starting with zero, signifying the lack of hypertension, and continuing with the different stages of hypertension. Current age and body mass index were treated as continuous variables. Numeric variables are presented as mean value \pm standard variation (SD) and compared by using IndependentSamples $t$-test. Descriptive statistics were done depending on two strata represented by the presence and absence of TDP. Dichotomized TDP was used in the majority of statistical analyses. According to the recommendations from other studies, the NA and SI subscales scores were treated as distinct ordinal variables only in the multiple linear regression model. ${ }^{26,27}$ The Chi-squared test was used to compare the frequency of nominal variables. For continuous variables, Independent-Samples $t$-test was applied to compare means and for ordinal variables Mann-Whitney U-test was carried out to analyze mean rank differences. Partial correlations were calculated to estimate the relationship between DS 14 subscales scores and the Syntax score after controlling for anxiety and depression levels. In addition, multiple linear regression was performed to estimate the association of cardiovascular morbidity risk factors such as the family history of cardiovascular diseases, body mass index, smoking, DM, current age, gender, dyslipidemia, hypertension, NA and SI (as distinct ordinal variables) with CAD complexity reflected by Syntax score. For the statistical analyses, we used the software package SPSS version 11.5 (SPSS Inc, 
Table I The Frequency of TDP and the Differences of Mean Ranks for Negative Affectivity and Social Inhibition Based on the Presence or Absence of Type D Personality

\begin{tabular}{|l|l|l|l|}
\hline Subjects Enrolled in Study, $\mathbf{n}(\%)$ & Presence of TDP, $\mathbf{n}(\%)$ & Absence of TDP, $\mathbf{n}(\%)$ & Significance of Differences \\
\hline $221(100 \%)$ & $42(19 \%)$ & $179(81 \%)$ & \\
\hline Negative affectivity & 169.17 & 97.35 & $\mathrm{P}<0.001$ \\
\hline Social inhibition & 197.37 & 90.73 & $\mathrm{P}<0.001$ \\
\hline
\end{tabular}

Notes: The level of significance for all analyses was set at $\alpha=0.05$; mean ranks are reported to either strata considered separately. Mann-Whitney $U$-test was performed. Abbreviation: TDP, type D personality.

Chicago, IL). A two-sided p-value of $<0.05$ was accepted as statistically significant.

\section{Results}

In our 221 consecutive hospitalized patients with chest pain (60 \pm 10.2 years; 131 men), referred for coronary angiography, the final diagnosis was: acute ST-segment elevation myocardial infarction in 61 patients $(27.6 \%)$, non-ST-elevation acute myocardial infarction in 25 patients $(11.3 \%)$, unstable angina in 45 patients $(20.3 \%)$ and stable angina associated with obstructive coronary artery disease in 51 patients (23\%). Other 39 patients presented non-obstructive coronary artery diseases (21.7\%) and $27(12.2 \%)$ had a previous coronary revascularization. In our group, 148 patients' beneficiated of PCI (66.9\%) and 34 of CABG (15.3\%). The mean Syntax score in our study group was $17.53 \pm 10.4$.

By applying the DS 14 scale we identified 42 (19\%) patients with TDP. There were significant differences between the mean ranks of NA (169.17 vs 97.35; $\mathrm{p}<0.001)$ and SI $(197.37$ vs $90.73 ; \mathrm{p}<0.001)$ depending on the two analyzed strata (Table 1).

The sociodemographic characteristics were mostly similar between the two considered strata, making them comparable in this regard (Table 2). There was only one statistically significant difference between the means of the current age of males vs females $(58.92 \pm 10.72$ vs $62.12 \pm 9.49, \mathrm{p}=0.023)$. Moreover, CAD patients with TDP have tended to reside in urban regions that are probably more demanding for daily existence compared to rural areas, therefore shaping the personality profile during their psychological development.

The psychoactive substance use has not been significantly different between the non-TDP and TDP in our CAD patients. Neither current smokers $(45.8 \%$ vs $52.4 \%$, respectively, $\mathrm{p}=0.443)$ nor current alcohol users $(27.4 \%$ vs $31.0 \%$, respectively, $\mathrm{p}=0.642$ ) made a difference between the compared strata.
The CAD appreciated by mean Syntax score was more complex in the subgroup of patients with TDP in comparison to non-TDP subgroup $(26.21 \pm 12.03$ vs $15.49 \pm 8.89$, respectively, $\mathrm{p}<0.001$ ) (Table 2).

We also analyzed the differences between the mean ranks of several distinct symptom dimensions of SCL-90 inventory depending upon the presence or absence of TDP, as shown in Table 3. Except for the obsessive-compulsive symptoms ( 126.73 vs 107.31 , respectively; $p=0.076$ ), the rest of SCL-90 symptom dimensions (somatization, interpersonal sensitivity, depression, anxiety, hostility, phobic anxiety, paranoid ideation and psychoticism) have significantly higher levels in the subgroup of TDP with CAD patients (all p < 0.05) (Table 3).

Given that, the higher level of psychopathological dimensions measured by the SCL-90 scale could have partially influenced the magnitude of the Syntax score, we calculated the partial correlations between the two dimensions of TDP with the Syntax scores after controlling for anxiety and depression scores as measured by the mentioned scale (Table 4).

Hence, both dimensions of TDP, NA ( $\mathrm{r}=0.178, \mathrm{p}=0.008$ and $\mathrm{r}=0.150, \mathrm{p}=0.026$, respectively) and SI $(\mathrm{r}=0.253$, $\mathrm{p}<0.008$ and $\mathrm{r}=0.183, \mathrm{p}=0.006$, respectively) have remained statistically significant, correlated with the Syntax scores indicating a direct contribution of them.

The presence of acute myocardial infarction was significantly higher in the subgroup of patients with TDP (57.1\%, 24 patients with TDP vs $34.6 \%, 62$ patients without TDP, $\mathrm{p}=0.007)$. Table 2 also showed a higher incidence of occluded coronary vessels in patients with TDP $(\mathrm{p}<0.001)$.

The value of the echocardiographic parameters is also influenced by the presence of TDP in CAD patients (Table 5). These patients have more altered systolic dysfunction [LVEF (47.45 $\pm 13.78 \%$ vs $51.7 \pm 9.48 \%$, p=0.06), S' $(7.05 \pm 2.57 \mathrm{~cm} / \mathrm{s}$ vs $8.89 \pm 3.06 \mathrm{~cm} / \mathrm{s}, \mathrm{p}<0.001)$ and global longitudinal strain 
Table 2 Sociodemographic, Case History and Clinical Data

\begin{tabular}{|c|c|c|c|}
\hline Sociodemographic and Anamnesis Data & Non-TDP CAD Patients & TDP CAD Patients & Statistical Significance \\
\hline n (\%) & 179 (8I\%) & $42(19 \%)$ & - \\
\hline Average current age, mean (SD) ${ }^{a}$ & $60.08(10.369)$ & $60.81(10.317)$ & $p=0.683$ \\
\hline Gender - male, n (\%) & 107 (59.8\%) & $24(57.1 \%)$ & $p=0.755$ \\
\hline Educational level, mean ranks & 111.56 & 108.60 & $p=0.772$ \\
\hline Residency - urban area, n (\%) & $110(61.5 \%)$ & $32(76.2 \%)$ & $p=0.073$ \\
\hline Professional status - Employed or Student, n (\%) & 57 (31.8\%) & 14 (33.3\%) & $p=0.852$ \\
\hline Marital status - with intimate partner, n (\%) & I 48 (82.7\%) & 37 (88.1\%) & $p=0.393$ \\
\hline Positive family history of CVD, n (\%) & $24(13.4 \%)$ & 7 (16.7\%) & $P=0.584$ \\
\hline Current smokers ${ }^{\mathrm{b}}, \mathrm{n}(\%)$ & $82(45.8 \%)$ & $22(52.4 \%)$ & $p=0.443$ \\
\hline Occasionally alcohol use (not abuse or dependence) ${ }^{\mathrm{b}}, \mathrm{n}(\%)$ & 49 (27.4\%) & $13(31.0 \%)$ & $P=0.642$ \\
\hline Syntax score, mean (S.D.) & I5.49 (8.892) & $26.21(12.038)$ & $P<0.001$ \\
\hline The presence of myocardial infarction, $n(\%)^{c}$ & $62(34.6 \%)$ & $24(57.1 \%)$ & $P=0.007$ \\
\hline No. of occluded coronary vessels, mean ranks ${ }^{c}$ & 102.87 & 145.67 & $\mathrm{P}<0.00 \mathrm{I}$ \\
\hline Staged hypertension, mean ranks & 108.90 & 119.96 & $p=0.199$ \\
\hline Dyslipidemia, n (\%) & $39(21.8 \%)$ & $6(14.3 \%)$ & $P=0.277$ \\
\hline Body mass index, mean (S.D.) & $26.113(3.1324)$ & $26.595(3.9177)$ & $p=0.394$ \\
\hline Preexisting diabetes mellitus irrespective of type, $n$ (\%) & 67 (37.4\%) & 14 (33.3\%) & $P=0.620$ \\
\hline
\end{tabular}

Notes: The level of significance for all analyses was set at $\alpha=0.05$; the listed percentages and means are reported to either strata considered separately. ${ }^{a}$ There was

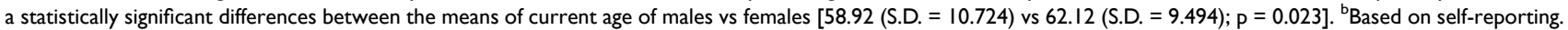
'Cardiac status at the admission in Cardiac Intensive Care Unit.

Abbreviations: CAD, coronary artery disease; CVD, cardiovascular disease; TDP, type D personality.

Table 3 The Differences in Symptomatology Profiles in CAD Patients Depending Upon the Presence or Absence of Type D Personality

\begin{tabular}{|l|l|l|l|}
\hline SCL-90 Domains of Symptomatology (Mean Ranks) & Non-TDP CAD Patients & TDP CAD Patients & Statistical Significance \\
\hline $\mathrm{n}(\%)$ & $179(81 \%)$ & $42(19 \%)$ & \\
\hline Somatization & 104.18 & 140.08 & $\mathrm{P}=0.00 \mathrm{I}$ \\
\hline Obsessive-compulsive & 107.31 & 126.73 & $\mathrm{P}=0.076$ \\
\hline Interpersonal sensitivity & 101.63 & 150.94 & $\mathrm{P}<0.00 \mathrm{I}$ \\
\hline Depression & 98.60 & 163.86 & $\mathrm{P}<0.001$ \\
\hline Anxiety & 102.74 & 146.19 & $\mathrm{P}<0.00 \mathrm{I}$ \\
\hline Hostility & 104.61 & 138.21 & $\mathrm{P}=0.002$ \\
\hline Phobic anxiety & 102.69 & 146.43 & $\mathrm{P}<0.00 \mathrm{I}$ \\
\hline Paranoid ideation & 102.41 & 147.60 & $\mathrm{P}<0.00 \mathrm{I}$ \\
\hline Psychoticism & 100.91 & 154.01 & $\mathrm{P}<0.00 \mathrm{I}$ \\
\hline
\end{tabular}

Notes: The level of significance for all analyses was set at $\alpha=0.05$; the listed percentages and means are reported to either strata considered separately. Mann-Whitney U-test was performed for all comparisons of SCL-90 domains of symptomatology.

Abbreviations: SCL-90, Symptom Check List - 90; CAD, coronary artery disease; TDP, type D personality. 
Table 4 The Partial Correlations Between the DS 14 Subscales Score and Syntax Score After Controlling for Anxiety and Depression Levels as Measured by SC-90 Scale

\begin{tabular}{|l|l|l|}
\hline \multirow{2}{*}{$\begin{array}{l}\text { DS I4 Subscales } \\
\text { Scores }\end{array}$} & Syntax Score \\
\cline { 2 - 3 } & $\begin{array}{l}\text { Controlling for Anxiety Levels as Measured on } \\
\text { SCL-90 Scale }\end{array}$ & $\begin{array}{l}\text { Controlling for Depression Levels as Measured on } \\
\text { SCL-90 Scale }\end{array}$ \\
\hline Negative affectivity & $r=0.178 ; p=0.008$ & $r=0.150 ; p=0.026$ \\
\hline Social inhibition & $r=0.253 ; p<0.001$ & $r=0.183 ; p=0.006$ \\
\hline
\end{tabular}

Abbreviations: DS 14, the 14-item Type D Scale; SCL-90, Symptom Check List - 90.

Table 5 Echocardiographic Parameters Depend on the Presence or Absence of TDP in CAD Patients

\begin{tabular}{|l|l|l|l|l|}
\hline \multicolumn{2}{|l|}{ Echocardiographic Parameters in CAD Subjects } & $\begin{array}{l}\text { CAD Patients with } \\
\text { TDP; Mean (S.D.) }\end{array}$ & $\begin{array}{l}\text { CAD Patients } \\
\text { without TDP; Mean } \\
\text { (S.D.) }\end{array}$ & $\begin{array}{l}\text { Significance of } \\
\text { Differences }\end{array}$ \\
\hline \multirow{3}{*}{ Systolic function } & Left ventricular ejection fraction (\%) ${ }^{\mathrm{a}}$ & $47.45(13.788)$ & $51.77(9.48 \mathrm{I})$ & $\mathrm{P}=0.060$ \\
\cline { 2 - 5 } & Peak systolic mitral A=annular velocity $(\mathrm{cm} / \mathrm{s})$ & $7.053(2.575)$ & $8.898(3.069)$ & $\mathrm{P}<0.001$ \\
\cline { 2 - 6 } & Global longitudinal strain $(\%)$ & $-14.067(5.650)$ & $-15.938(4.822)$ & $\mathrm{P}=0.030$ \\
\hline \multirow{2}{*}{ Diastolic function } & $\begin{array}{l}\text { The early diastolic transmitral velocity/early mitral } \\
\text { annular diastolic velocity ratio (E/E') }\end{array}$ & $13.498(4.155)$ & $10.242(3.254)$ & $\mathrm{P}<0.001$ \\
\cline { 2 - 5 } & Left atrial volume $(\mathrm{mL})^{\mathrm{a}}$ & $85.79(34.436)$ & $71.03(26.497)$ & $\mathrm{P}=0.012$ \\
\hline
\end{tabular}

Notes: The level of significance for all analyses was set at $\alpha=0.05$; The listed means are reported to either strata considered separately. ${ }^{a}$ Levene's Test for Equality of Variances statistically significant.

Abbreviations: CAD, coronary artery disease, S.D., standard deviation; TDP, type D personality.

$(-14.06 \pm 5.65 \%$ vs $-15.93 \pm 4.82 \%, \mathrm{p}<0.001)]$, more altered LV diastolic function assessed by $\mathrm{E} / \mathrm{E}$ ' index (13.49 $\pm 4.15 \mathrm{vs}$ $10.24 \pm 3.25, \mathrm{p}=0.03)$ and larger LAV $(85.79 \pm 34.4 \mathrm{~mL}$ vs $71.03 \pm 26.49 \mathrm{~mL}, \mathrm{p}=0.012$ ) (Table 5).

The number of occluded coronary vessels and the number of patients with myocardial infarction were significantly higher represented in CAD patients with TDP. They are quite intriguing results, even more so because the major cardiovascular risk factors (heredity, smoking, staged hypertension, dyslipidemia, DM) did not differ between the two compared strata (Table 2).

Table 6 outlines the contributing role of TDP and risk factors for CAD to the Syntax score.

Male gender, age, BMI, heredity, hypertension did not reach a significant value in our study group (all $\mathrm{p}>0.05$ ), while smoking $(\beta=0.132, \quad \mathrm{p}=0.037), \quad$ dyslipidemia $(\beta=0.149, \quad p=0.013), \quad$ Diabetes Mellitus $\quad(\beta=232$, $\mathrm{p}<0.001)$, NA dimension of TDP $(\beta=0.255, \mathrm{p}<0.001)$ and SI $(\beta=0.279, p<0.001)$ dimension of TDP have a significant contribution to the complexity of CAD assessed by Syntax score.

\section{Discussion}

In the present study, we analyzed the relationship between TDP and CAD complexity assessed by Syntax score in patients referred for coronary angiography. We demonstrate for the first time a more complex CAD in patients with TDP, irrespective of the anxiety and depression symptoms influences.

To date, numerous studies have revealed different psychological paradigms, including behavioral patterns, underpinning the connection between the TDP and the severity of CAD disease. ${ }^{8,9,28-30}$ Our results are inconsistent with some previous studies, in which such a relationship was not traced. Probably, the reason is that in previous studies homogeneous groups of patients were examined (before $\mathrm{CABG}, \mathrm{PCI}$, during cardiac rehabilitation, etc.), and in the present study, the surveyed cohort is heterogeneous. We included all the patients with chest pain referred for coronary angiography after noninvasive evaluation. For diagnostic cath procedures, $12.5 \%$ of patients had a prior coronary revascularization, in comparison with $25 \%$ as reported in the CathPCI Registry. ${ }^{31}$ In 
Table 6 The Contributing Role of TDP and Risk Factors for CAD to the Syntax Score

\begin{tabular}{|l|l|l|l|l|}
\hline Risk Factors & Beta Standardized Coefficient & SE & p value & $\mathbf{9 5 \%}$ C.I. for B Unstandardized Coefficient \\
\hline Male gender & -0.104 & 1.333 & 0.100 & $-4.829-0.425$ \\
\hline Current age & 0.114 & 0.062 & 0.065 & $-0.007-0.237$ \\
\hline Body Mass Index & -0.027 & 0.183 & 0.640 & $-0.447-0.275$ \\
\hline Smoking & 0.132 & 1.317 & 0.037 & $0.166-5.357$ \\
\hline Positive family history of CVD & 0.027 & 1.758 & 0.641 & $-2.645-4.286$ \\
\hline Hypertension (staged) & 0.026 & 0.676 & 0.665 & $-1.039-1.625$ \\
\hline Dyslipidemia & 0.149 & 1.527 & 0.013 & $0.834-6.855$ \\
\hline Preexisting Diabetes Mellitus (DM) & 0.232 & 1.290 & $<0.001$ & $2.467-7.552$ \\
\hline NA dimension of type D personality & 0.255 & 0.112 & $<0.001$ & $0.213-0.655$ \\
\hline SI dimension of type D personality & 0.279 & 0.147 & $<0.001$ & $0.346-0.924$ \\
\hline
\end{tabular}

Notes: Model summary (adjusted R square $=0.286 ; \mathrm{R}$ square change $=0.318$; $\mathrm{F}$ change $=9.809 ;$ significant $\mathrm{F}$ change $<0.00 \mathrm{I}$ ).

Abbreviations: SE, standard error; CVD, cardiovascular disease; NA, negative affectivity; SI, social inhibition; DM, diabetes mellitus, irrespective of type; TDP, type D personality.

our series $27.6 \%$ patients presented acute ST-segment elevation myocardial infarction (versus $16 \%$ in CathPCI Registry), $11.3 \%$ non-ST-elevation acute myocardial infarction (versus $9 \%$ in CathPCI Registry), 20.3\% unstable angina (33\% in CathPCI Registry) and 23\% stable angina (21\% in CathPCI Registry). The percent of patients with non-obstructive coronary artery diseases was similar with this one from CathPCI Registry $(21.7 \%$ vs $18 \%)$.

\section{Behavioral Patterns}

It has been shown that the TDP measured by using the DS 14 scale was significantly associated with cardiovascular diseases. ${ }^{8,9}$ However, the increasing comorbidity of major and minor depression with cardiovascular diseases has provided for sufficient research to investigate the mediating effect of depression on this category of physical diseases in individuals having a TDP. ${ }^{14,32-34}$ In a previous study, TDP was correlated in patients with CAD with more unhealthy behaviors and less healthy behaviors, while controlling for anxiety and depression. These results are in line with our findings according to which TDP in CAD patients have had slightly more frequent addictive behaviors such as nicotine and alcohol use compared to nonTDP subset of CAD patients. Moreover, TDP may be closely related to the unfavorable evolution of CAD by engaging other unhealthy behaviors, such as low adherence to treatment, propensity for dysfunctional coping styles and resistance to lifestyle changing or post-event cardiac rehabilitation. ${ }^{12,30,35,36}$

At the same time, the TDP has been studied concerning its capacity to exert a negative effect on the quality of life in cardiovascular patients. Indeed, the TDP may impair health-related quality of life in CAD patients by mediating the role of anxiety and depressive overlapping symptoms. ${ }^{30}$ Thus, one study has found that TDP was significantly associated with poor self-perceived symptoms in patients with mild coronary abnormalities, which can be supposed to be deleterious over the long term. ${ }^{35-40}$ A dyadic cross-sectional study has revealed that the TDP and perceived control were closely related to the healthrelated quality of life in both cardiac arrest survivors and their spouses. ${ }^{41}$ The social support and coping style were shown to be hypothetically underlying mechanisms that can, in part, explain the relationships between TDP and physical symptoms, and TDP and perceived stress, respectively. $^{42}$

In our study, the percent of CAD patients that fulfilled the criteria for TDP was similar to those found in other research, meaning that about one in five patients has the pre-existing maladaptive personality traits. ${ }^{27,28}$ The sociodemographic characteristics were mostly similar between the two considered strata, making them comparable in this regard. However, CAD patients with TDP have tended to reside in urban regions that are probably more demanding for daily existence compared to rural areas, therefore 
shaping the personality profile during their psychological development. Other large representative research has found that different types of personality disorders, classified according to DSM III - R, were more frequent in the urban areas than living on the outskirts. ${ }^{43}$

\section{Psychopathological Aspects}

Concerning the higher levels of general psychopathological dimensions that we have found in CAD patients with TDP, including depression and anxiety, these findings have already been evidenced in other researches. ${ }^{30,32,33}$ Therefore, the assumption that TDP negatively impacts the health outcomes of CAD patients, not singularly, but in a broader context of psychosocial factors clustered within the same individual is quite feasible. ${ }^{36}$ Nonetheless, considering our results even after controlling for anxiety and depressive levels, the TDP has been significantly associated with the complexity of CAD assessed by Syntax score. On the upside, the propensity for maladaptive coping styles that have been documented by other researches, in conjunction with higher levels of psychopathologies, might support our result whereby the perceived stressful life events are significantly more self-reported by TDP in CAD patients in contrast to non-TDP controls. ${ }^{35,44}$ It was the only anamnesis data revealed by our research that made the difference between the two compared subsets of CAD patients.

Regarding the negative influences of TDP on the cardiac status of CAD patients, several overlapped psychophysiological mechanisms may support this negative outcome. Given its innate component represented by temperament, which is mainly originating in cerebral neurotransmission profile, TDP may continuously alter the homeostasis of specific physiological parameters. ${ }^{45}$ Intermittently, against the background of the continuous influence, the physiological imbalances can be amplified by the interposing of the anxiety and depressive symptoms, which in turn, are promoted by the presence of TDP. $^{36}$ Therefore, the mind-heart interactions may be influenced by several neuroendocrine and neurobiological pathways such as the hypothalamic-pituitary-adrenal and sympathoadrenal medullary axes dysfunction, chronic low-grade inflammation, endothelial cell dysfunction, neurotransmitters imbalances, altered level of neurotrophic factors and the dysconnectivity of cortico-subcortical neural networks. ${ }^{46,47}$ Higher comorbidity between distinct physical illnesses such as dyslipidemia, hypertension, DM, and obesity, and CAD and stress-related conditions, including TDP, may also be impacted by the abovementioned pathophysiological processes.

\section{Biological Substrate}

From the biological standpoint, the TDP stems from the imbalance of the cerebral neurotransmitters reflected by altered levels of specific metabolites, such as tryptophan metabolite kynurenine. ${ }^{13}$ It has been noticed that restricted sleep mediates the connection between TDP and a low or blunted cardiovascular response to the social stressors, increasing the risk for a range of adverse health outcomes even in healthy individuals. ${ }^{14,15}$ Other research data have shown that TDP is more associated with endothelial dysfunction and inflammation plasma biomarkers. ${ }^{16-18}$ Also, some studies have indirectly tied the TDP to coronary heart diseases through the intermediate action of dyslipidemia. ${ }^{19-21}$

TDP individuals may be subject to a biased interpretation of their health status dues to dysfunctional cognitive schemas that underlay the negative affective experiences through the erroneous interpretation of objective reality, leading to a more amplified negative affectivity and autonomic over activity. ${ }^{48}$ In a clinical study, the presence of TDP was independently associated with the occurrence of ventricular arrhythmias as a marker of sympathetic dysregulation. ${ }^{49}$ In both healthy individuals and CAD patients, the presence of TDP has been associated with increased diurnal cortisol release. ${ }^{50,51}$

Intriguing results were attained regarding the current or past myocardial infarction and the number of occluded coronary vessels that were significantly higher represented in CAD patients with TDP. The more so because all the major risk factors like heredity, smoking, staged hypertension, dyslipidemia, body mass index, and DM did not differ between the two compared strata. Recently, research have repeatedly evidenced that atherosclerosis, especially $\mathrm{CAD}$, are no longer considered as a simple lipid storage disorder. These might also be considered chronic inflammatory diseases, where increased levels of TNF- $\alpha$, other cytokines and increased IFN- $\gamma$ transcription factors will lead to endothelial cell dysfunction and atherosclerotic plaques formation. ${ }^{52-55}$

Besides cytokines, the serum high-sensitivity C-reactive protein has been found as a highly predictive factor of cardiovascular risk, irrespective of the lowdensity lipoprotein cholesterol level. ${ }^{56}$ Low-grade chronic inflammation intercedes in all phases of the atherosclerosis process, starting with the accumulation of macrophage transformed into foam cells in the arterial wall continuing 
with the fatty streak and fibrous plaque formation and ending with plaque fissuring, rupture, and thrombosis, all leading to an acute coronary event. It has been shown that atherosclerosis is accompanied by an excessive inflammatory response resulting in fibroproliferative reaction at the level of subintimal arterial space promoting subsequent thrombus formation as a direct consequence of various forms of injuries leading to an acute cardiac event. ${ }^{57,58}$ Furthermore, traditional major cardiovascular risk factors (eg, smoking, hypertension, hyperglycemia, obesity or insulin resistance), together with stress-related psychopathological conditions, have the propensity to induce chronic inflammation resulting in endothelial activation through impaired nitric oxide production and loss of vasodilator and antithrombotic properties of the coronary endothelium. ${ }^{57,58}$ Similarly, research has revealed that the heart is an active endocrine organ and a series of endocrine molecules (generically named cardiokines) are released by cardiomyocytes, fibroblasts, vascular endothelial cardiac cells and immune cells that reside in the heart. ${ }^{59}$ Moreover, the immune functions at the heart level are also regulated by the autonomous nervous system through the neuro-cardiac axis by its two components. The sympathoadrenal axis stimulates immunological response mainly by the effect of NE on resident macrophages in addition to that on circulating monocytes. In opposite, the parasympathetic nerves mediate acetylcholine release both in the reticuloendothelial system and in the heart, where the neurotransmitter binds the nicotinic receptor expressed on resident cardiac macrophages, blocking the release of the main proinflammatory cytokines (ie, TNF-a, IL-1) ${ }^{60}$ The TDP and CAD may partially share the same cascade of overactivated pro-inflammatory cytokines alongside the imbalanced functioning of sympathetic and parasympathetic, respectively, components of the autonomous nervous system. Altogether, these biological mechanisms may result in a worsened prognosis of the TDP CAD patients. ${ }^{9}$

It seems that specific and subtle immunological pathways can connect the stress-related disorders, such as depression, anxiety, or even negative affectivity component of TDP with cardiovascular diseases.

All converging biological and psychological mechanisms mentioned above have determined that TDP, alongside the major depression, has been included in the European Cardiovascular Prevention guideline as a risk factor to screen for atherosclerosis. ${ }^{61,62}$ Based on a holistic perspective, the mixture of physical and psychological manifestations of CAD could be entirely integrated into a systemic nosological concept where TDP is one of the stable parts of a whole syndrome entity. In these particular cases, amid the chronic manifestations of CAD patients, acute exacerbations periodically occur in both planes, psychologically mainly expressed as contextual symptoms of anxiety and depression, and physically marked by biological deteriorations of the cardiac parameters. Therefore, TDP should be considered as a critical psychological marker of a more unfavorable clinical course in CAD patients, characterized by a higher likelihood of negative outcomes and cardiac events and a poorer quality of life compared to their non-TDP counterparts.

Our results should be considered in the context of several limitations. The study population of patients undergoing coronary angiography was heterogeneous. Since this study is a cross-sectional design, the direction of the mediation between TDP and depressive symptoms cannot be confirmed. Despite the good response rate (95.2\%), the non-consenting patients may have refused consent because of a higher level of depressive symptoms, leading to some bias in the results. This work was performed on a relatively small group of patients, and does not provide longer-term follow-up of the clinical implications of TDP. It is a single center study and its reproduction in other centers or by multicenter studies could support its validity.

\section{Conclusion}

In patients referred for coronary angiography, TDP was associated with a more complex CAD assessed by Syntax score. Given its stability, TDP may be viewed as a dynamic interface between the underlying biological and psychological vulnerabilities and the bodily and psychological symptoms of CAD.

In other news, an interdisciplinary and personalized based therapeutic approach of patients suffering from $\mathrm{CAD}$ is more than necessary considering the psychological profile of each patient. Beyond the quality of life improvement, in well-defined cases, the psychological intervention, at times, combined with psychopharmacological treatment might result in extending the survival period in CAD patients with TDP by targeting the altered psychophysiological pathways. Further larger and prospective studies, including more biological, clinical and anamnestic data, are absolutely required.

\section{Disclosure}

The authors report no conflicts of interest in this work. 


\section{References}

1. Lozano R, Naghavi M, Foreman K, et al. Global and regional mortality from 235 causes of death for 20 age groups in 1990 and 2010: a systematic analysis for the Global Burden of Disease Study 2010. Lancet. 2012;380:2095-2128. doi:10.1016/S0140-6736(12)61728-0

2. Townsend N, Nichols M, Scarborough P, et al. Cardiovascular disease in Europe - epidemiological update 2015. Eur Heart J. 2015;36:696-705. doi:10.1093/eurheartj/ehv428

3. Lichtman JH, Froelicher ES, Blumenthal JA, et al; American Heart Association Statistics Committee of the Council on Epidemiology and Prevention and the Council on Cardiovascular and Stroke Nursing. Depression as a risk factor for poor prognosis among patients with acute coronary syndrome: systematic review and recommendations: a scientific statement from the American Heart Association. Circulation. 2014;129:1350-1369. doi:10.1161/ CIR.0000000000000019

4. MacKenzie G, Greig M, Hay I, et al. Competing risk analysis of factors related to long-term incidence of CHD. J Epidemiol Community Health. 2017;71:33-36. doi:10.1136/jech-2016-207347

5. Akhabue E, Thiboutot J, Cheng JW, et al. New and emerging risk factors for coronary heart disease. Am J Med Sci. 2014;347:151-158. doi:10.1097/MAJ.0b013e31828aab45

6. Friedman M, Rosenman RH. Association of specific overt behavior pattern with blood and cardiovascular findings; blood cholesterol level, blood clotting time, incidence of arcus senilis, and clinical CAD. J Am Med Assoc. 1959;69:1286-1296. doi:10.1001/ jama.1959.03000290012005

7. Pah AM, Bucuras P, Buleu F, et al. The importance of DS-14 and HADS questionnaires in quantifying psychological stress in type 2 diabetes mellitus. Medicina. 2019;55:569. doi:10.3390/ medicina55090569

8. Williams L, O'Connor RC, Grubb NR, et al. TDP and illness perceptions in myocardial infarction patients. $J$ Psychosom Res. 2011;70:141-144. doi:10.1016/j.jpsychores.2010.07.015

9. O’Dell KR, Masters KS, Spielmans GI, et al. Does type D personality predict outcomes among patients with cardiovascular disease? A meta-analytic review. $J$ Psychosom Res. 2011;71:199-206. doi:10.1016/j.jpsychores.2011.01.009

10. Bekendam MT, Kop WJ, Barzilay S, et al. The predictive value of positive affect and Type D personality for adverse cardiovascular clinical outcomes in patients with non-obstructive coronary artery disease. J Psychosom Res. 2018;104:108-114. doi:10.1016/j. jpsychores.2017.11.003

11. Meyer T, Hussein S, Lange HW, Herrmann-Lingen C. Type $\mathrm{D}$ personality is unrelated to major adverse cardiovascular events in patients with coronary artery disease treated by intracoronary stenting. Ann Behav Med. 2014;48(2):156-162. doi:10.1007/s12160014-9590-2

12. Arnett DK, Blumenthal RS, Albert MA, et al. ACC/AHA Guideline on the primary prevention of cardiovascular disease: a report of the American College of Cardiology/American Heart Association task force on clinical practice guidelines. Circulation. 2019;140:e596e646. doi:10.1161/CIR.0000000000000678

13. Dietary Guidelines Advisory Committee. Dietary Guidelines for Americans 2015-2020. 8th ed. Washington, DC: U.S: Department of Health and Human Services and U.S. Department of Agriculture; December 2015 [Internet]. Available from: http://health.gov/dietary guidelines/2015/guidelines. Accessed June 10, 2020.

14. Lang RM, Badano LP, Mor-Avi V, et al. Recommendations for cardiac chamber quantification by echocardiography in adults: an update from the American Society of Echocardiography and the European Association of Cardiovascular Imaging. Eur Heart $J$ Cardiovasc Imaging. 2015;16:233-270. doi:10.1093/ehjci/ jev014
15. Quiñones MA, Otto CM, Stoddard M, et al; Doppler Quantification Task Force of the Nomenclature and Standards Committee of the American Society of Echocardiography. Recommendations for quantification of doppler echocardiography: a report from the doppler quantification task force of the nomenclature and Standards Committee of the American Society of Echocardiography. J Am Soc Echocardiogr. 2002;15:167-184. doi:10.1067/mje.2002.120202

16. Nagueh SF, Middleton KJ, Kopelen HA, et al. Doppler tissue imaging: a noninvasive technique for evaluation of left ventricular relaxation and estimation of filling pressures. $\mathrm{J} \mathrm{Am} \mathrm{Coll} \mathrm{Cardiol.}$ 1997;30:1527-1533. doi:10.1016/S0735-1097(97)00344-6

17. Ommen SR, Nishimura RA, Appleton CP, et al. Clinical utility of Doppler echocardiography and tissue Doppler imaging in the estimation of left ventricular filling pressures: a comparative simultaneous Doppler-catheterization study. Circulation. 2000;102:1788-1794. doi:10.1161/01.CIR.102.15.1788

18. Smiseth OA, Torp H, Opdahl A, et al. Myocardial strain imaging: how useful is it clinical decision making? Eur Heart J. 2016;37:1196-1207. doi:10.1093/eurheartj/ehv529

19. Reisner SA, Lysyansky P, Agmon Y, et al. Global longitudinal strain: a novel index of left ventricular systolic function. $J \mathrm{Am}$ Soc Echocardiogr. 2004;17:630-633. doi:10.1016/j.echo.2004.02.011

20. Franzone A, Taniwaki M, Rigamonti F, et al. Angiographic complexity of CAD according to SYNTAX score and clinical outcomes after revascularisation with newer-generation drug-eluting stents: a substudy of the BIOSCIENCE trial. EuroIntervention. 2016;12: e595-604. doi:10.4244/EIJV12I5A99

21. Bundhun PK, Sookharee Y, Bholee A, et al. Application of the SYNTAX score in interventional cardiology: a systematic review and meta-analysis. Medicine. 2017;96:e7410. doi:10.1097/MD.0000000000007410

22. Denollet J. DS14: standard assessment of negative affectivity, social inhibition, and Type D personality. Psychosom Med. 2005;67:89-97. doi:10.1097/01.psy.0000149256.81953.49

23. Emons WH, Meijer RR, Denollet J. Negative affectivity and social inhibition in cardiovascular disease: evaluating type D personality and its assessment using item response theory. J Psychosom Res. 2007;63:27-39. doi:10.1016/j.jpsychores.2007.03.010

24. Kupper N, Pedersen SS, Höfer S, et al. Cross-cultural analysis of type $\mathrm{D}$ (distressed) personality in 6222 patients with ischemic heart disease: a study from the International HeartQoL Project. Int $J$ Cardiol. 2013;166:327-333.

25. Derogatis LR, Savitz KL. The SCL-90-R and the Brief Symptom Inventory (BSI) in Primary Care. In: Maruish ME, Mahwah NJ, editors. Handbook of Psychological Assessment in Primary Care Settings. 2nd ed. Lawrence Erlbaum Associates; 2000:297-334.

26. Coyne JC, Jaarsma T, Luttik ML, et al. Lack of prognostic value of TDP for mortality in a large sample of heart failure patients. Psychosom Med. 2011;73:557-562. doi:10.1097/PSY.0b013e318227ac75

27. Williams L, O'Connor RC, Grubb N, et al. TDP predicts poor medication adherence in myocardial infarction patients. Psychol Health. 2011;26:703-712. doi:10.1080/08870446.2010.488265

28. Mols F, Martens EJ, Denollet J. TDP and depressive symptoms are independent predictors of impaired health status following acute myocardial infarction. Heart. 2010;27:52-58.

29. Bunevicius A, Brozaitiene J, Staniute M, et al. Decreased physical effort, fatigue, and mental distress in patients with CAD: importance of personality-related differences. Int $J$ Behavioral Med. 2014;21:240-247. doi:10.1007/s12529-013-9299-9

30. Al-Qezweny MN, Utens EM, Dulfer K, et al. The association between type D personality, and depression and anxiety ten years after PCI. Neth Hear J. 2016;24:538-543. doi:10.1007/s12471-016-0860-4

31. Dehmer GJ, Weaver D, Roe MT, et al. A contemporary view of diagnostic cardiac catheterization and percutaneous coronary intervention in the United States: a report from the CathPCI Registry of the National Cardiovascular Data Registry, 2010 through June 2011. J Am Coll Cardiol. 2012;60(20):2017-2031. doi:10.1016/j.jacc.2012.08.966 
32. Starrenburg AH, Kraaier K, Pedersen SS, et al. Association of psychiatric history and TDP with symptoms of anxiety, depression, and health status prior to ICD implantation. Int $J$ Behav Med. 2013;20:425-433. doi:10.1007/s12529-012-9244-3

33. Lambertus F, Herrmann-Lingen C, Fritzsche K, et al. Prevalence of mental disorders among depressed coronary patients with and without Type D personality. Results of the multi-center SPIRR-CAD trial. Gen Hosp Psychiatry. 2018;50:69-75. doi:10.1016/j.genhosppsych.2017.10.001

34. Doi-Kanno M, Fukahori H. Predictors of depression in patients diagnosed with myocardial infarction after undergoing percutaneous coronary intervention: a literature review. $J$ Med Dent Sci. 2016;63:37-43. doi:10.11480/jmds.630301

35. Borkoles E, Kaiseler M, Evans A, et al. Type D personality, stress, coping and performance on a novel sport task. PLoS One. 2018;13: e0196692. doi:10.1371/journal.pone.0196692

36. Kupper N, Denollet J. TDP as a risk factor in coronary heart disease: a review of current evidence. Curr Cardiol Rep. 2018;20:104. doi:10.1007/s11886-018-1048-x

37. Kwon M, Kang J. Mediating effect of illness perception on the relationship between TDP and health behaviors among CAD patients. Health Psychol Open. 2018;5:2055102918817228. doi:10.1177/2055102918817228

38. Williams L, Wingate A. Type D personality, physical symptoms and subjective stress: the mediating effects of coping and social support. Psychol Health. 2012;27:1075-1085. doi:10.1080/ 08870446.2012 .667098

39. Ginting H, van de Ven M, Becker ES, et al. TDP is associated with health behaviors and perceived social support in individuals with coronary heart disease. $J$ Health Psychol. 2016;21:727-737. doi:10.1177/1359105314536750

40. Mommersteeg PM, Pot I, Aarnoudse W, et al. TDP and patient-perceived health in nonsignificant CAD: the TWeesteden mIld STenosis (TWIST) study. Qual Life Res. 2013;22:2041-2050. doi:10.1007/s11136-012-0340-2

41. Israelsson J, Persson C, Bremer A. et al. Dyadic effects of TDP and perceived control on health-related quality of life in cardiac arrest survivors and their spouses using the actor-partner interdependence model. Eur J Cardiovasc Nurs;2019. 1474515119890466 doi:10.1177/1474515119890466

42. Staniute M, Brozaitiene J, Burkauskas J, et al. Type D personality, mental distress, social support and health-related quality of life in CAD patients with heart failure: a longitudinal observational study. Health Qual Life Outcomes. 2015;13:1. doi:10.1186/s12955-014-0204-2

43. Torgersen S, Kringlen E, Cramer V. The prevalence of personality disorders in a community sample. Arch Gen Psychiatry. 2001;58:590-596. doi:10.1001/archpsyc.58.6.590

44. Booth L, Williams L. TDP and dietary intake: the mediating effects of coping style. J Health Psychol. 2015;20:921-927. doi:10.1177/ 1359105315573433

45. Cloninger CR, Cloninger KM, Zwir I, et al. The complex genetics and biology of human temperament: a review of traditional concepts in relation to new molecular findings. Transl Psychiatry. 2019;9:290. doi:10.1038/s41398-019-0621-4

46. Mayberg HS. Positron emission tomography imaging in depression: a neural systems perspective. Neuroimaging Clin $N \mathrm{Am}$. 2003;13:805-815. doi:10.1016/S1052-5149(03)00104-7
47. Dean J, Keshavan M. The neurobiology of depression: an integrated view. Asian J Psychiatr. 2017;27:101-111. doi:10.1016/j.ajp.2017.01.025

48. Howard S, O'Riordan A, Nolan M. Cognitive bias of interpretation in Type D personality: associations with physiological indices of arousal. Appl Psychophysiol Biofeedback. 2018;43:193-201. doi:10.1007/s10484-018-9397-1

49. Einvik G, Dammen T, Namtvedt SK, et al. TDP is associated with increased prevalence of ventricular arrhythmias in community residing persons without coronary heart disease. Eur J Prev Cardiol. 2014;21:592-600. doi:10.1177/2047487312462800

50. Bibbey A, Carroll D, Ginty AT, et al. Cardiovascular and cortisol reactions to acute psychological stress under conditions of high versus low social evaluative threat: associations with the TDP construct. Psychosom Med. 2015;77:599-608. doi:10.1097/ PSY.0000000000000194

51. Molloy GJ, Perkins-Porras L, Strike PC, et al. TDP and cortisol in survivors of acute coronary syndrome. Psychosom Med. 2008;70:863-868. doi:10.1097/PSY.0b013e3181842e0c

52. Libby P. The molecular mechanisms of the thrombotic complications of atherosclerosis. J Intern Med. 2008;63:517-527. doi:10.1111/ j.1365-2796.2008.01965.x

53. Uyemura K, Demer LL, Castle SC, et al. Cross-regulatory roles of interleukin (IL)-12 and IL-10 in atherosclerosis. J Clin Invest. 1996;97:2130-2138. doi:10.1172/JCI118650

54. Methe H, Brunner S, Wiegand D, et al. Enhanced T-helper-1 lymphocyte activation patterns in acute coronary syndromes. $\mathrm{J}$ Am Coll Cardiol. 2005;45:1939-1945. doi:10.1016/j.jacc.2005.03.040

55. Fioranelli M, Bottaccioli AG, Bottaccioli F, et al. Stress and inflammation in CAD: a review psychoneuroendocrineimmunology-based. Front Immunol. 2018;9:2031. doi:10.3389/fimmu.2018.02031

56. Ridker PM, Danielson E, Fonseca FA, et al. Rosuvastatin to prevent vascular events in men and women with elevated c-reactive protein N Engl J Med. 2008;359:2195-2207. doi:10.1056/NEJMoa0807646

57. Li JJ. Inflammation in CADs. Chin Med J. 2011;124:3568-3575.

58. Libby P. Inflammation and cardiovascular disease mechanisms. Am J Clin Nutr. 2006;83:456S-460S. doi:10.1093/ajen/83.2.456S

59. Masayuki S, Noriyuki O, Kenneth W. Cardiokines: recent progress in elucidating the cardiac secretome. Circulation. 2012;26:e327-332.

60. Borovikova LV, Ivanova S, Zhang M, et al. Vagus nerve stimulation attenuates the systemic inflammatory' response to endotoxin. Nature. 2000;405:458-462. doi:10.1038/35013070

61. Piepoli MF, Hoes AW, Agewall S, et al. European Guidelines on cardiovascular disease prevention in clinical practice: the Sixth Joint Task Force of the European Society of Cardiology and Other Societies on Cardiovascular Disease Prevention in Clinical Practice (constituted by representatives of 10 societies and by invited experts) Developed with the special contribution of the European Association for Cardiovascular Prevention \& Rehabilitation (EACPR). Eur Heart Journal. 2016;37:2315-2381.

62. Ageu LŞ, Levai CM, Andreescu NI, et al. Modern molecular study of weight gain related to antidepressant treatment: clinical implications of the pharmacogenetic testing. Rom $J$ Morphol Embryol. 2018;59:165-173. 


\section{Publish your work in this journal}

Neuropsychiatric Disease and Treatment is an international, peerreviewed journal of clinical therapeutics and pharmacology focusing on concise rapid reporting of clinical or pre-clinical studies on a range of neuropsychiatric and neurological disorders. This journal is indexed on PubMed Central, the 'PsycINFO' database and CAS, and is the official journal of The International Neuropsychiatric Association (INA). The manuscript management system is completely online and includes a very quick and fair peer-review system, which is all easy to use. Visit http://www.dovepress.com/testimonials.php to read real quotes from published authors.

Submit your manuscript here: https://www.dovepress.com/neuropsychiatric-disease-and-treatment-journal 